\title{
Anniversary of Nikolai Nikolaevich Bogolyubov
}

PACS numbers: $01.60 .+\mathrm{q}$

DOI: $10.1134 / \mathrm{S} 1063779609040066$

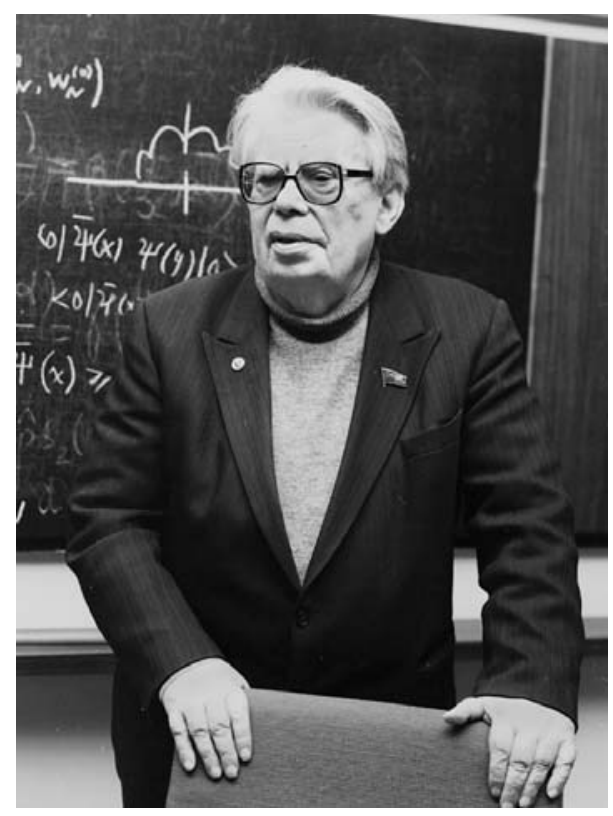

21st, August 2009 will mark the 100th anniversary of a great Russian scientist, Academician Nikolai Nikolaevich Bogolyubov.

The worldwide community recognizes the outstanding and often fundamental contribution of Academician Bogolyubov to developing an impressive number of fields in mathematics, mechanics, statistical physics, quantum field theory, and elementary particle physics.

The unique characteristic of the scientific style of Bogolyubov has consisted in the capability to evaluate the core of a problem and to intuitively envisage its principal solvability even in cases when known methods left no hope for it. He was a Master in Bulgakov's sense of the word.

Bogolyubov was heading the Joint Institute for Nuclear Research in Dubna for almost 25 years (19651989). It is under his leadership that the JINR turned into one of the leading nuclear physics centers of the world.

Nikolai Nikolaevich devoted much attention to the training of young scientists. He is credited for creating world-renowned scientific schools, such as a school of mathematical physics and non-linear mechanics in Kiev, school of theoretical and mathematical physics in Moscow and Dubna.

He was a founder of our journal "Physics of Particles and Nuclei" and its first editor-in-chief in the period 1969-1992.

Bogolyubov was elected a foreign member of many academies abroad and was an honorary doctor of a number of the most prestigious universities of the world.

In December 2008 the President of Russia D. A. Medvedev and President of Ukraine V. A. Yushchenko nearly simultaneously signed the decrees on the celebration of the 100th anniversary of Academician Nikolai Nikolaevich Bogolyubov in Russia and Ukraine, respectively. According to these decrees, a number of official Anniversary celebrations and international conferences will take place in Moscow, Dubna, and Kiev. 